\title{
Excitation and destruction of nuclei in hot astrophysical plasmas around black holes
}

\section{Ervin Kafexhiu*}

Max Planck Institut für Kernphysik

E-mail: ervin.kafexhiu@mpi-hd.mpg.de

\section{Gabriela S. Vila}

Instituto Argentino de Radioastronomía (IAR - CONICET)

E-mail: gvila@iar-conicet.gov.ar

\section{Felix Aharonian}

Dublin Institute for Advanced Studies \& Max Planck Institut für Kernphysik

E-mail: felix.aharonian@dias.ie

The importance of nuclear reactions in low-density astrophysical plasmas with ion temperatures $T \geq 10^{10} \mathrm{~K}$ has been recognized for thirty years. However, the lack of comprehensive data banks of relevant nuclear reactions and the limited computational power have not previously allowed detailed theoretical studies. Recent developments in these areas make it timely to conduct comprehensive studies of the nuclear properties of very hot plasmas formed around compact relativistic objects such as black holes and neutron stars. Such studies are of great interest in the context of scientific programs of future low-energy cosmic gamma-ray spectrometry. In this work, using the publicly available code TALYS, we have built a large nuclear network relevant for temperatures exceeding $10^{10} \mathrm{~K}$. We then study the evolution of hot accretion plasmas for such high temperatures due to the destruction of nuclei at inelastic collisions, calculate the resulting gamma-ray spectra due to the superposition of prompt excitation gamma-ray lines, and present the results on the abundances of $\mathrm{D}, \mathrm{T},{ }^{3} \mathrm{He}$.

11th Symposium on Nuclei in the Cosmos, NIC XI

July 19-23, 2010

Heidelberg, Germany

\footnotetext{
${ }^{*}$ Poster presenter.
} 


\section{Introduction}

Low-density optically thin, hot plasmas with ion temperature exceeding $1 \mathrm{MeV}$ can be formed in different astrophysical environments, in particular, in accretion flows close to black holes and in strong shock waves related, for example, to supernova explosions. The most straightforward approach to studying the dynamics of formation and evolution of such plasmas is the detection of prompt $\gamma$-ray lines which are unambiguous signatures of specific nuclei.

Collisions in thermal plasmas with temperatures exceeding $1 \mathrm{MeV}$ are characterized not only by excitation reactions with emission of gamma-ray lines, but also by the destruction of nuclei. In such plasmas, the destruction of nuclei proceeds on timescales shorter than excitation times. Thus, only prompt de-excitation lines related to specific nuclei are expected to contribute to the plasma radiation [2]. The presence of a given prompt gamma-ray line is related to the existence of a given nucleus, which is itself governed by the destruction processes. This suggests that there should exist a strong relation between the gamma-ray line spectrum and nuclear destruction processes. The break-up processes are sensitive to the plasma temperature and density; therefore, the detection of gamma-ray lines and their spectral profile evolution may reveal unique information about the chemical composition and physical parameters of the plasma, such as temperature and density at any given time.

The first attempts to assess the importance of nuclear processes in hot, thin astrophysical plasmas were made in $[1,2]$. There is estimated the contribution of gamma-ray line emission to the luminosity of spherically symmetric accretion flows and accretion disks. In these early works, however, only a limited number of reactions could be taken into account, mainly due to the lack of data on nuclear cross sections and the increased computational complexity of calculations as more nuclei are considered.

Most of these difficulties have nowadays been overcome. The introduction of on-line nuclear databases $[3,4]$ provides easy access to experimental and theoretical data on nuclear cross sections. There also exist publicly available computer codes that allow the calculation of nuclear cross sections and other quantities of interest. These make use of several theoretical models.

In this work we calculate the temporal evolution of the composition and the gamma-ray line luminosity of a hot plasma with ion temperature $T_{\mathrm{i}} \geq 1 \mathrm{MeV}$ due to nuclear reactions. Although we presently concentrate on the radiative output, the study of nuclear reactions in hot plasmas may have other relevant applications, such as calculating the production of deuterium nuclei and its associated gamma-ray line in a non-cosmological way. The production of neutrons is also of considerable interest, since these particles can escape from the source and interact with nearby targets.

\section{Cross Sections}

Any detailed treatment of the processes described above requires complete information about the cross sections of all the relevant interactions, which are necessary to calculate the correspondent reaction rates. Presently, there are several publicly available codes which allow calculation of these cross-sections. In this work we have performed our calculations using TALYS - a convenient, multi-model code which offers optimized model parameters for reactions with target mass number 
$A>6$ [5]. For targets with $A \leq 6$, we have used ENDF evaluations or EXFOR experimental data to reconstruct cross sections as a function of projectile energy $[3,4]$. The cross-sections of some important reactions are shown in Fig. 2.
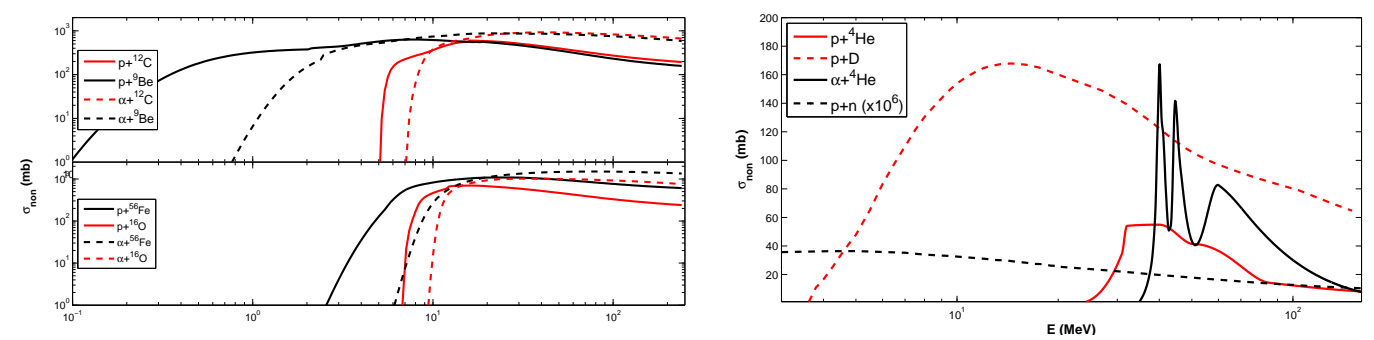

Figure 1: The total nonelastic cross sections as a function of the projectile energy for some relevant processes.

\section{Method and Calculations}

Presently, we have only considered reactions with protons $(p)$ and $\alpha$ particles as projectiles; this is a good approximation for plasmas with a standard cosmic composition [6]. Furthermore, we assumed that all nuclei are instantaneously in thermal equilibrium at the same temperature $T$, that they are all in the ground state, and that both the temperature and the total plasma density are constant in time. To calculate the composition evolution we basically have to solve the Boltzmann equation for every nuclear species in the plasma. The basic ingredient in these equations is the Maxwellian average $R(T)=\langle\sigma v\rangle$, where $\sigma$ is the cross section and $v$ the relative velocity between projectile and target; $R(T)$ is called the reaction rate. Some typical rates $R(T)$ are shown in Fig. 2.
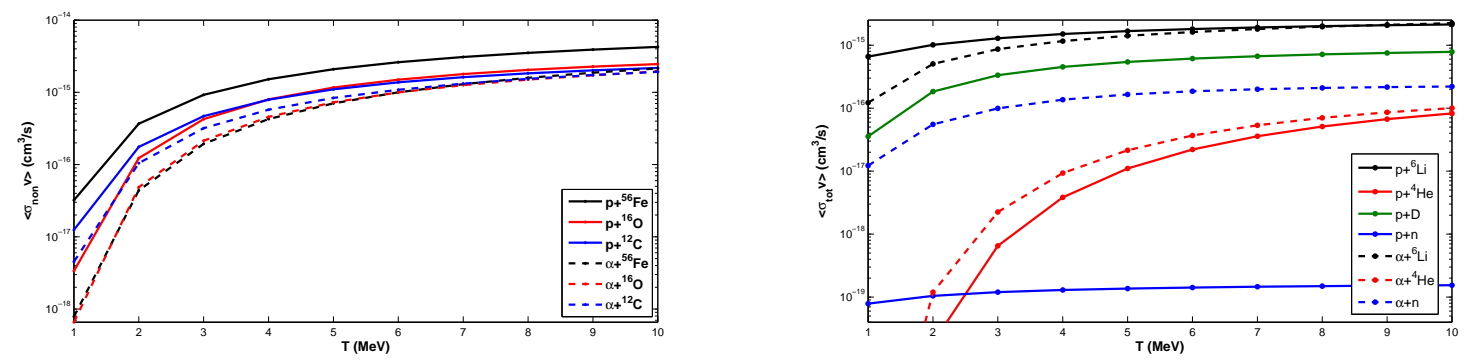

Figure 2: The rates of some typical reactions $<\sigma_{\text {non }} v>$.

We want to build a closed reaction network where every reaction will produce nuclei which are again in the network. For this purpose we introduced a threshold value for the reaction rate, $R^{\text {th }}=10^{-18} \mathrm{~cm}^{3} \mathrm{~s}^{-1}$, selecting only those channels which have $R(T)>R^{\text {th }}$. By making $R^{\text {th }}$ smaller the solutions get more accurate, but at the same time this increases the number of channels into consideration, therefore dramatically increasing the computational time. 
We start with all cosmic isotopes from ${ }^{6} \mathrm{Li}$ to ${ }^{70} \mathrm{Zn}$. Using TALYS we calculate their reaction cross sections with $p$ and $\alpha$ projectiles. Using these cross sections, we calculate the reaction rates for every channel and gamma-ray line for every target and projectile, and then filter the channels with rates larger than $R^{\text {th }}$. From the selected channels we may get some new nuclei. For these nuclei we follow the same procedure described above, until no new nuclei appear from any reaction. At this point the network is closed. For the elements with $A<6$ we used cross sections taken from evaluations performed, for example, by ENDF-VII.0, and experimental data. This network is valid for temperatures 1-10 MeV. Finally, having chosen a set of physical conditions for the plasma and its initial composition, we proceed to solve the network.

\section{Discussion}

We validated our network by calculating the mass abundance evolution of all the nuclei in a simple case. The initial mass fractions are $X_{p}=70 \%, X_{\alpha}=25 \%$, and $X_{{ }^{12} \mathrm{C}}=5 \%$, and the total nucleon number density is $\rho=10^{18} \mathrm{~cm}^{-3}$. We solved the network for two different temperatures, $T=1 \mathrm{MeV}$ and $T=3 \mathrm{MeV}$.

Figure 3 shows the temporal evolution of the number density for some specific nuclei and the emissivity of the plasma in gamma-ray lines. The value of the temperature has a strong impact on the characteristic timescales. The rates of almost all reactions increase with temperature, and correspondingly the abundances of nuclei and the gamma-ray emissivities evolve much faster. Note from Fig. 3 that some intermediate elements (result of break-up processes) become more abundant in certain periods of the evolution. At higher temperatures more interaction channels are opened, which leads to more $\gamma$-ray lines due to the de-excitation of nuclei. For temperatures $T \geq 1 \mathrm{MeV}$ the plasma quite quickly destroys all heavier nuclei, and the final state contains just protons and neutrons and some small traces of Deuterium and ${ }^{3} \mathrm{He}$, result of break-up processes and weak fusion reactions which take place on larger time scales. At this stage the gamma-ray emission almost disappears.

With this simple example we demonstrate the power of our large network of nuclear reactions for $T \geq 1 \mathrm{MeV}$ and the ability of the method to solve this network to a specified accuracy, by taking into account a large number of reactions and their resulting channels. Future work will consist of solving the network for realistic assumptions concerning the temporal and spatial dependence of the density and temperature realized in specific astrophysical environments, e.g. accretion flows in the vicinity of black holes and in strong shock waves related to supernova explosions.

\section{References}

[1] F.A. Aharonian \& R.A. Sunyaev, Gamma-ray line emission, nuclear destruction and neutron production in hot astrophysical plasmas. The deuterium boiler as a gamma-ray source, Mon. Not. R. Astr. Soc. 210257 (1984)

[2] F.A. Aharonian \& R.A. Sunyaev, Synthesis of light nuclei in a two-temperature astrophysical plasma, Astrofizika 27131 (1987)

[3] NDS-IAEA, Nuclear Data Service, International Atomic Energy Agency, http://www-nds.iaea.org/ 

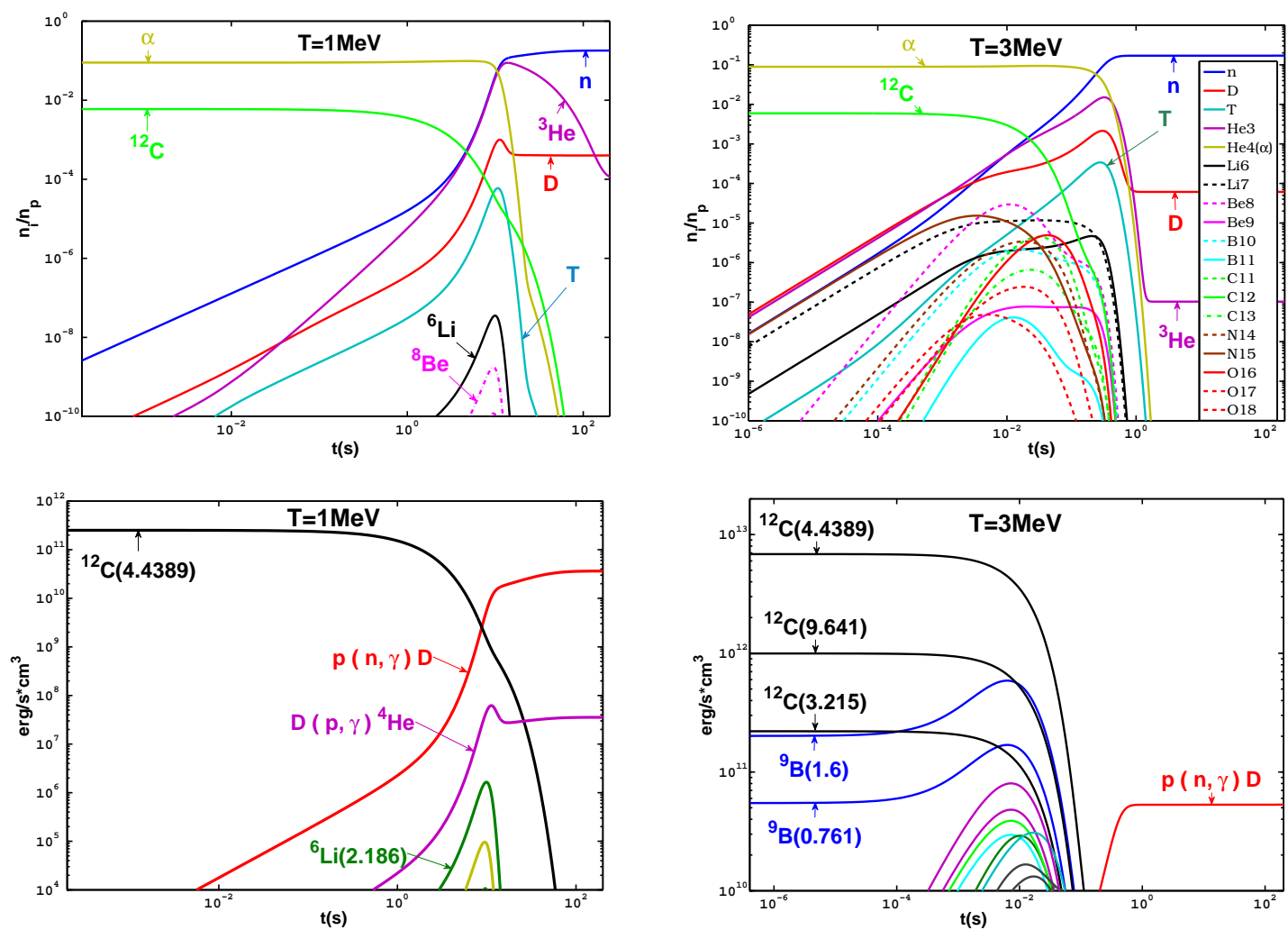

Figure 3: Temporal evolution of the fractional abundance $\left(n_{i} / n_{p}\right)$ for some specific network's nuclei and the plasma emissivity in gamma-ray lines, calculated for temperatures $T=1 \mathrm{MeV}$ and $T=3 \mathrm{MeV}$.

[4] NNDC-BNL, National Nuclear Data Center, Brookhaven National Laboratory, http://www.nndc.bnl.gov/

[5] TALYS code, Arjan Koning, Stephane Hilaire, Marieke Duijvestijn, http://www.talys.eu

[6] H.E. Suess \& H.C. Urey, Abundances of the Elements, Rev. Mod. Phys. 28, 53-74 (1956) 